Research Article

\title{
Sports and Health Management Using Big Data Based on Voice Feature Processing and Internet of Things
}

\author{
Lina Sun (iD) and Mingzhi Li \\ North China Institute of Science and Technology, Langfang, Hebei 065201, China \\ Correspondence should be addressed to Lina Sun; sunlina@ncist.edu.cn
}

Received 6 July 2021; Revised 10 August 2021; Accepted 12 August 2021; Published 26 August 2021

Academic Editor: Mian Ahmad Jan

Copyright ( $\odot 2021$ Lina Sun and Mingzhi Li. This is an open access article distributed under the Creative Commons Attribution License, which permits unrestricted use, distribution, and reproduction in any medium, provided the original work is properly cited.

\begin{abstract}
With the support of big data and information technology, various sectors such as sports, health, and medical industry can realize the integration and readjustment of the existing resources, which improve the operation efficiency of the industry and tap its huge potential. With the advancement in big data analysis, voice features, and Internet of Things (IoT), personalized health management is becoming the development trend and breakthrough of sports and health industry. The application of big data will tap out the huge potential of the sports and health industry. In this paper, we have used the Mel-requency cepstrum coefficient as the speech feature processing method. When the linear frequency is transformed to the Mel frequency by Fourier transform, the calculation accuracy will decrease with the increase in the frequency, and the low-frequency signal will be retained to improve the anti-noise ability. With further study of the voice feature processing and IoT model of big data's sports and health management, a vector addition regression was developed to compare the two real scoring features of the processing results that pave the way for further analysis and result evaluation. Through experimental verification, it is proved that the method in this paper can better learn the speech features. At the same time, with the introduction of noise reduction, the big data of speech recognition in sports health management has a stronger robustness and improves the overall system performance.
\end{abstract}

\section{Introduction}

In the education and teaching reforms, there is a lack of scientific and effective information management platform for physical health. In terms of processing and analysis of physical measurement of data, it fails to realize the professional, scientific, and dynamic development of the physical health information management system. Although relevant departments of education are required to collect the physical test data (from primary school to university graduates) every year, there is a lack of systematic and continuous management of the test data, and the efficiency of physical test is relatively low. In view of the fact that the decline of physical health has not been effectively curbed at present, we urgently need to establish a set of individualized and guidance system about physical health management and scientific exercise.

The development of the sports health management system aims at the comprehensive evaluation of physical test results by allowing a comprehensive and accurate understanding of the athlete's health level. Relying on the data in the database to make a detailed classification of physical conditions, the system can provide a more comprehensive system evaluation and more appropriate fitness guidance program for better exercise. After each stage of the test, the system modifies the user's system status according to the difference between the actual physical changes and the expected changes and accordingly modifies the exercise prescription for the next stage. Based on the physical test data and various physical indicators of colleges, a standard database is established $[1,2]$. The database not only can help us to check system status but also a detailed classification and effective fitness guidance scheme is given. Since the database is covering a wide range of data, it can help in our effective evaluation system in regional differences and the differences between schools, which can be more targeted to carry out sports activities [3]. Health management can draw lessons 
from a mental model of society, and at the same time, using the related theories and methods of management, it guides people to improve the life style, form good health habits, and improve the level of people's health. Thus, it helps to improve the quality of life of people, so it is necessary for the government to formulate a series of activities in a planned and organized system. It is not a school or a group that can handle individual behaviors $[4,5]$. Health management services must abide by the principle of standardization [6]. Standardization is the foundation of health management. If the cause of health management is to be fully developed, it is impossible to solve some urgent problems such as reducing the incidence of diseases and saving medical costs by relying only on existing conditions. Therefore, it is necessary to draw on effective experience [7]. The development of health management is bound to be benign. Under the guidance and planning of relevant institutions and in the formulation and implementation of government policies and regulations, it is entirely possible for us to establish a strict supervision and management mechanism to create a good atmosphere of health management for everyone [8].

Sports health industry is playing an increasingly important role in the economic development. Sports health industry and other industries have a very strong correlation, in their own development, and at the same time, one can also pull and promote the development of other industries, to provide the all-round development of the economy of power [9]. Of course, the expansion of the sports and health industry will have a greater demand for the number of employees, which will play a positive role in resolving the regional employment problem. This industry itself is the result of the sports industry and health industry's mutual confluence and has a very strong correlation in this new era [10]. The most typical is the sports competitions held allows for the huge promotion that has a significant effect on regional economic development. For example, the Soccer World Cup, an international sports event, needs to improve the infrastructure and increase the construction of venues. At the same time, it also plays a positive role in the local catering and accommodation industry, cultural tourism industry, and lottery industry $[11,12]$. Similarly, the health industry, especially the sports and health industry, is closely related to the pharmaceutical industry. Sports rehabilitation and physical fitness measurement are both part of the pharmaceutical industry, and the development of the sports and health industry will naturally promote the development of the pharmaceutical industry [13].

This pattern matching method has been adopted by most speech recognition research institutions. In the 1980s, a major breakthrough was made in acoustic model and speech model. The application of HMM to the acoustic model is an important development stage of speech recognition [14], and the acoustic model based on deep learning as the core has brought a significant improvement in speech recognition rate. In terms of feature extraction, linear predictive analysis [15], perceptual linear prediction coefficient [16], Mel-frequency cepstrum coefficient [17], and FBank feature based on filter banks are carried out frequently [18]. In terms of the language model, recursive big data sports health management, conditional random field, and other new modeling languages are used [19]. In the application of deep learning, the excitation function of hidden layer node is modified and applied to music processing. A multilevel conditional random field is applied to language recognition. Big data sports and health management are directly applied to the HMM state output modeling [20], which reduces the error rate compared with the traditional acoustic model. The current popular deep learning is integrated into the recommender system to study how to integrate multivariate data under big data and build a more appropriate user model to improve the performance of the recommender system [20]. The hybrid recommendation algorithm based on feature and close neighbors was studied by the authors. They combined the matrix algorithm and the collaborative filtering recommendation system based on the project ALS recommendation system, which was based on the matrix decomposition algorithm. The two platforms in the SPARK were executed to test the system $[21,22]$ and improve the scalability of the collaborative filtering recommendation system [23] and accuracy. The improved system filtering algorithm based on singular decomposition and the improved term-based algorithm were studied, which effectively improved the precision and quality of the recommendation system [24]. The personalized recommendation algorithm based on multiple interests of users is studied, which can deal with the personalized recommendation problem under multiple interests of users [25]. The conditional speech feature processing and the hidden factor model of the Internet of Things (IoT) used for personalized recommendation were studied, which can effectively extract features and can improve the recommendation hit rate of $3.11 \%$ compared with the general big data sports and health management system filtering $[26,27]$.

There are a series of problems in the development of sports and health industry, such as the lack of industry management standardization system, the development mode of sports and health industry is too vague, the lack of professional fitness and health guidance personnel, and low efficiency of publicity, respectively. Affected by the traditional employment environment, the field of sports and health service industry lacks professional technical personnel and management personnel. With the deepening of Internet technology into the economic system, the application of voice feature processing, IoT, and big data technology is necessary; otherwise, it will not be conducive to the sustainable development of the sports and health industry. The combination of sports and health industry and the Internet is a new blue ocean for the joint development of traditional entity industry and e-commerce industry at the present stage. By referring to physique and health assessment indicators, the individual indicators (including individual characteristics analysis, individual dynamic analysis, and correlation analysis of various indicators) are analyzed through big data in this paper. The system carries out complex calculation according to the latest test results of individual and their history, realizes intelligent analysis and calculation through the situation simulator, and provides various forms of result analysis reports. At the same time, the 
system refers to the evaluation standard to develop tailormade exercise methods. Our system presents these methods to the Internet. On the one hand, the use of the physique and health system can improve sports enthusiasm. It can also provide the most efficient and scientific guidance methods so as to achieve the purpose of improving physical health level.

The rest of this paper is organized as follows. In Section 2, speech feature processing and big data-enabled sports health management using the Internet of things is discussed. This section is our main work that highlights the significance of this paper. In Section 3, the experimental results and analysis are provided. Finally, the paper is concluded in Section 4.

\section{Speech Feature Processing and Big Data- Enabled Sports Health Management Using Internet of Things}

In this section, first we discuss the sports health framework which is based on big data analysis. Next, we discuss the voice feature processing and Internet of Things (IoT) for sports health research using big data.

2.1. Big Data-Enabled Sports Health Framework. The original intention of the big data physical health research framework is to promote enthusiasm for exercise, improve physical quality, and provide personalized guidance. Therefore, it is necessary to provide functions that can be accessed independently, rather than only those that are provided solely by the teachers. While using the big data sports health management and fitness guidance system, people only need to input their own personal information to get the relevant data of the physical test at any time. Changing the past physical test data is only in the hands of the test teacher or test department, and they do not understand their own test results that lack the longitudinal and horizontal data comparison phenomenon. Artificial intelligence and physique and health management system can be modified according to individual cervix information matching case in the database that will be the most closest to the truth and then try to analyze the individual status. Thus, the most personalized assessment results will be available that give the customized exercise programs. These results can eventually adjust the exercises according to the guidelines. At any stage, one can exercise the assessment result after transferring to the system that analyzes the latest data according to the physical conditions of the current and changed physical phase difference, to modify the next phase of the training plan. Thus, improvement scheme for the exercise can be provided according to the latest plan to exercise so as to promote the improvement of sports health management level with big data. Its frame diagram is shown in Figure 1.

Setting up the scientific and reliable evaluation standard is the key of the big sports health management system. It is the foundation of the whole system that is as realistic as possible the standardized norm and as far as possible detailed analysis of the physical condition of an individual. This will require a large and diverse data for the support, from low level to high level, so as to form a relatively complete system. Big data used by the sports and fitness guidance system for the management of health management method of collecting data is the first step. We can access the data in sports health management to understand the system parameters. Also, the personal information collected from historical data of the cervix form the basis of our data set. The physical measurement information and physical information in the data set are classified, and each kind of physical condition is matched with the required exercise program, so as to obtain a complete training prescription design process and a big data set of scientific guidance programs, which are our evaluation criteria.

Through a systematic process, the different functions of each department are standardized in operation logic, which are mainly reflected in the following steps:

(1) Collection and Documentation of Health Data. This is the first step in health management. Only by collecting all personal health information and understanding their health status, we can effectively maintain health. Our approach adds the personal information in a database by collecting the data from the school health management and physical examination sections. Moreover, a fitness test is conducted every semester in the school hospital and mental health center by collecting the medical record that includes mental health counseling questionnaires regarding the physical health data aggregation and health management system. From the perspective of the school, this system is conducive to enhance the interaction of various departments related to health and evaluate physical health in an all-round and multiangle way. From the perspective of $s$, it is necessary to ensure that are familiar with their own health conditions from a more professional and authoritative perspective and pay more attention to their own health.

(2) Health Status Analysis and Assessment. Sports activities according to the individual cervix grades for planning to exercise analysis, to the individuals school hospital records and cervix, combining analysis of evaluation of sports exercise and the feasibility of the project, prescribing a movement, from the perspective of medical health further exercise provide the basis and also provide important reference for physical education teachers teaching arrangement. Using the health management model for comprehensive professional analysis, we evaluate the health status based on an evaluation index to establish the corresponding health grade and further improve their roles for better understanding. This is actually the second step of health management to assess the risk of a disease. If the individuals are aware of the health risks, they will voluntarily rectify their unhealthy behaviors. On this basis, schools can make personalized health management plans, which provide an effective channel for communication between health management departments and individuals. 

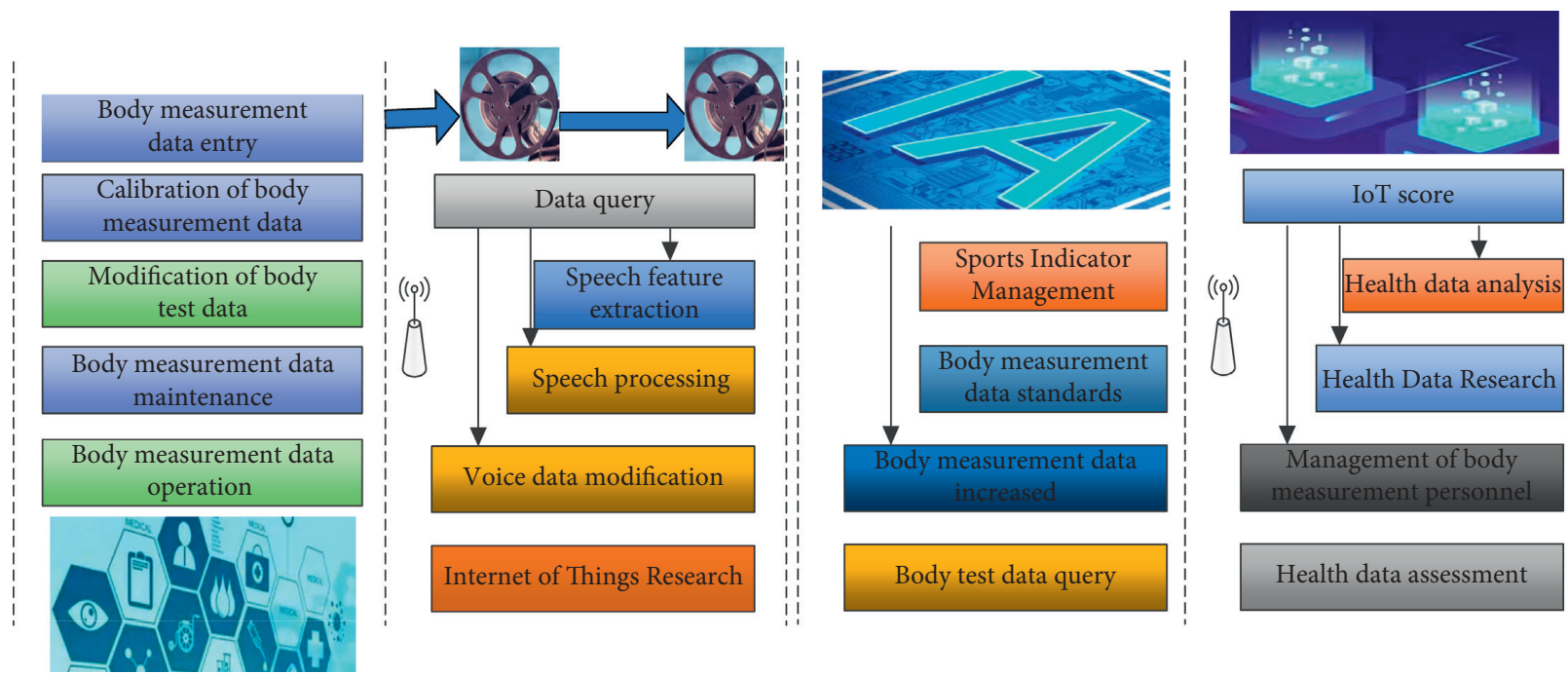

FIGURE 1: Sports and health framework of big data based on speech feature processing and the IoT.

2.2. Voice Feature Processing and Internet of Things for Sports Health Research with Big Data. After speech signal preprocessing, it is necessary to reduce the influence of irrelevant information and reduce the amount of data for subsequent speech recognition. Therefore, it is necessary to carry out feature extraction for speech signal. The feature extraction method adopted in this paper is the Mel-frequency cepstrum coefficient. The frequency of human speech signal is low, most of the information is contained in the low-frequency part, and the high frequency signal is susceptible to noise interference. MFCC transforms the linear frequency to the Mel-frequency through Fourier transform. Since the calculation accuracy of MFCC will decrease with the increase in frequency, the low-frequency signal will be retained to improve the antinoise ability. The study found that the human ear hears the sound frequency below $1 \mathrm{kHz}$, the volume increases with the increase in the frequency, and a linear relationship above $1 \mathrm{kHz}$ is a logarithmic relationship. According to this characteristic, MFCC found the correspondence between the actual frequency of speech and Mel frequency:

$$
F_{\text {Mrht }}(x)=1150 \ln \left(\frac{1+x}{800}\right) .
$$

Extracting the phonetic characteristics of automatic encoder can guarantee good noise robustness, a variation of the automatic encoder structure noise reduction automatic encoder, random erase part of the original input matrix, man-made noise, formation damage data, by fully training speech samples, and susceptibility to particular intelligent household voice control instructions. It greatly increases the accuracy of speech feature extraction. During the training of the autoencoder, in order to make the hidden layer learn more representative characteristics, it is necessary to restore the original data from the noise data so that the autoencoder must conduct noise reduction processing. The extraction flow chart of MFCC voice feature parameters is shown in Figure 2.
The specific steps are as follows:

(a) The speech spectrum FFT is obtained by fast Fourier transform. The preprocessed speech signal of each frame is shown as follows:

$$
x_{n}(t)=\sum_{m} x_{n}(m) e^{-u(m) / N^{N}} .
$$

(b) Calculate the Spectral Line Energy. The energy of the spectrum is calculated after FFT transformation, as shown in the following:

$$
E_{n}(x)=X_{n}(t) X_{n}^{T}(t)
$$

(c) Calculate the Mel Filter Energy. Each frame is passed through the filter in turn, and its energy in the Mel filter is calculated. The energy spectrum $E(K)$ of each frame is multiplied by the Mel filter energy, and finally the products are added up.

$$
S_{n}(m)=\sum_{t=0} E_{n}(x) H_{m}(x) .
$$

(d) Calculate the MFCC Coefficient. The logarithmic power spectrum is obtained by logarithmic operation of the filter energy, and then the MFCC coefficient is obtained by cosine transformation:

$$
C_{n}(x)=\sum_{m=1}^{M} \log S_{n}(m) \cos \frac{p t(x-0.5)}{m}
$$

Table 1 shows the experimental environment for the development and training of the recommendation algorithm model of sports and health management based on the big data of voice feature processing and the Internet of Things.

The development of the physical health management system aims at the comprehensive evaluation of the results of the physical test so as to have a more comprehensive and accurate understanding of their own health level. By making 



FIGURE 2: MFCC speech feature parameter extraction process.

TABLE 1: Experimental development environment.

\begin{tabular}{lc}
\hline Experimental environment & Detailed configuration \\
\hline Operating system & Windows 10 \\
Processor & i5, 3.0 GHz \\
A programming language & Python3.7 \\
Programming development environment & Jupyter notebook \\
Deep learning framework & TensorFlow2.0 \\
\hline
\end{tabular}

a detailed classification of the physical condition based on the data in the database, the system can provide a more comprehensive system evaluation and a more appropriate fitness guidance program to guide better exercise. After each stage test, the system will modify the user's system status according to the difference between the actual physical changes and the expected changes and accordingly modify the exercise prescription for the next stage.

A system standard database is established based on the physical test data and various physical indexes of colleges and universities. The database not only helps us to make a detailed classification and effective fitness guidance system but at the same time it covers a wide range of data that allows us to effectively evaluate the system based on regional differences among the schools. In our study, the database is targeted more toward sports activities.

Individual indicators (including individual characteristics analysis, individual dynamic analysis, and correlation analysis of various indicators) were analyzed by referring to physical fitness and health assessment indicators through big data. The system carries out complex calculation according to the latest and historical test results of the individual, realizes intelligent analysis and calculation through the situation simulator, and provides various forms of result analysis reports. At the same time, according to the evaluation standard, a tailored exercise method is developed through calculation. Our system presents these methods over the network. On the one hand, the use of the constitution and health system can improve the enthusiasm of sports; on the other hand, it can also provide the most efficient and scientific guidance methods so as to achieve the purpose of improving the level of physical health. The application of big data in the physical health management system has four advantages: high accuracy, good personalization, timeliness and high efficiency.

By further studying the encoding types of fields in these datasets, it can be found that some fields belong to the same category in the dataset. The usual method of data set processing is to encode these category fields into one hot encoding, but category fields like UserID and FoodID will be transformed into very sparse matrix. There is a phenomenon that the encoding dimension of the input field expands rapidly. This situation should be avoided. Therefore, the algorithm model is shown in Figure 3 in this paper. A vector addition regression was made by comparing the results with 


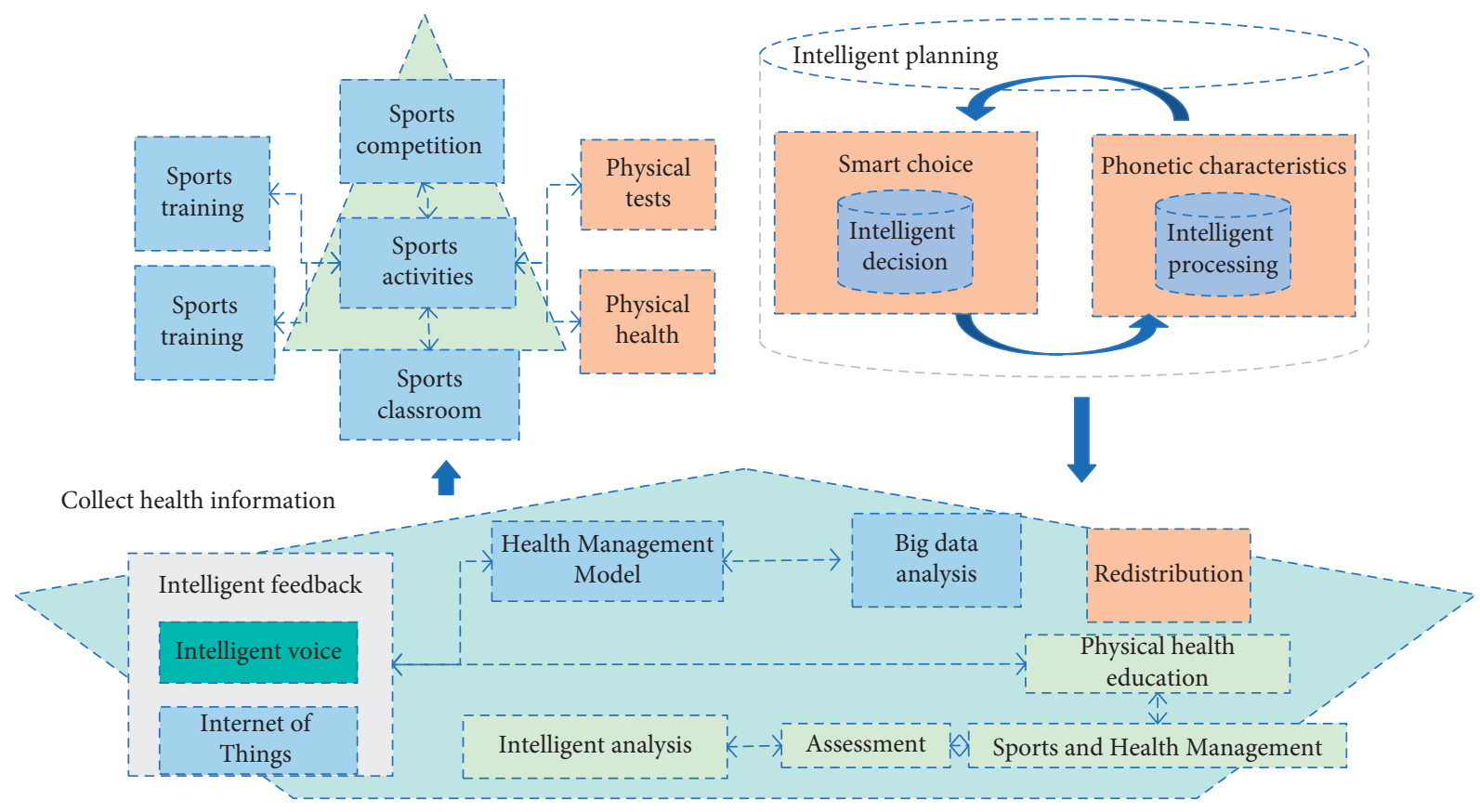

FIGURE 3: Big data sports and health management algorithm.

the real results of the two scoring features, and the scoring loss was optimized by the MSE method.

Based on the age group, the data of each stage and the ratio of male and female were obtained, and the number and proportion of this age group were quickly found out by the way of age input. The personnel data of each stage are shown in Table 2.

The original intention of the research on sports and health management with big data is to promote enthusiasm for exercise, improve physical quality, and provide personalized guidance. Therefore, it is necessary to provide with rights and functions that can be accessed independently, instead of only teachers having access. When using the big data sports health management and fitness guidance system, they only need to input their own personal information to get the relevant data of the physical test at any time. Changing the past physical test data is only in the hands of the test teacher or test department, and they do not understand their own test results that lack the longitudinal and horizontal data comparison phenomenon. The artificial intelligence and physique and health management system can be modified according to individual cervix information matching case in the database that will be the most close to the truth and then try to analyze the individual status and thus the most personalized assessment results will be available that gives the corresponding exercise program and can eventually take exercise according to the guidelines. At each stage, we can evaluate the assessment results after transfer to the system by analyzing the latest data. According to the physical condition of the existing phase difference, we can modify the next phase of the training plan to provide an improved scheme for exercise, which is in accordance with the latest plan to promote the improvement of sports health management level using big data. Through this system,
Table 2: Personnel data of each stage.

\begin{tabular}{lc}
\hline Age & Person \\
\hline 1995 & 105 \\
1996 & 194 \\
1997 & 104 \\
1998 & 135 \\
1999 & 124 \\
2000 & 146 \\
\hline
\end{tabular}

teachers can count overall sports and health management level with big data and put forward their own sports concepts in the system to understand the significance and suggestions of sports. In addition, teachers can also publish offline tasks in the system to urge to do physical exercises.

\section{Example Verification and Experimental Results}

In this paper, the usual performance, the duration of motor skill intervention learning, and the duration of exercise were taken as input indexes, and the changes in assessment performance, psychological cognition, health knowledge cognition, and various physical measurement results were taken as output indexes. According to the established inputoutput index system, the data are further processed as follows. Data processing of changes in the cognitive level of health knowledge is as follows: according to the scores of health cognition level measured before and after the experiment, the degree of changes in the cognitive level of health knowledge after a semester of study can be obtained. The logarithmic function is used to process changes in the cognitive level of health knowledge. Data processing of changes in psychological cognitive level is as follows: the 
innovation of this paper lies in the evaluation of mental health cognitive level. Lower the score of psychological state and psychological cognitive level, lower will be the score. The inverse function is used to evaluate changes in the overall psychological cognitive level. As an example, Table 3 provides the sample of data processing.

Comparative experiments in this paper aim to build the phonetic characteristics of 6 implicit layer processing and large data of IoT-enabled sports health management. The extraction of speech signal feature is converted to a 96-dimensional vector with the help of normalized processing and hidden layer activation function using Sigmoid function. The bBatch gradient descent method is used to cumulate values, with the initial vector of 0.03 . Because of big data sports health management, increasing the number of nodes is simple than increasing hidden layer units and can effectively improve the recognition. Hence, in this paper, the phonetic characteristics of processing large data of Internet of Things sport health management are studied by adding varying number of hidden layer nodes. In this work, we conducted the study with contrast experiment on the number of hidden layer nodes. The experiment was conducted for different values of $n$ such as 10,20,30, 40, 50, and 100 , with different iterations; loss value is calculated after each iteration is completed, and recognition accuracy is calculated every 10 iterations. Speech feature processing and large data of Internet of things sports health management under different hidden layer node number change curve, and the identification accuracy loss value curve is as shown in Figures 4 and 5; these figures show that with an increase in the number of nodes, the training time and speech recognition accuracy increase. However, after node number 30 , the training time increases with the increase in the number of nodes rapidly. Therefore, in deep voice feature processing and big data sports and health management of the Internet of Things, the network performance is the best when the number of hidden layer nodes is 30 .

Initialize network parameters according to denoising the autoencoder model. Some network node values were randomly set to 0 to simulate the noise. The initial values of RBM model parameters were obtained by using the random function 0.0006 rand () to get a small value. The bias was set to 0 , the learning rate was set to 0.003 , and the learning rate of the link weight between hidden layers was set to 0.006 . After the training of each layer of the network model is completed, the Softmax activation function is used to finetune the network. The maximum number of iterations is set to be 100 , and the iteration is stopped when the change rate of the mean square error of the results of two adjacent iterations is less than 0.002. As shown in Figures 6 and 7, the change curve of loss value of three different autoencoders and the recognition accuracy curve show that the 5-layer depth autoencoder has a better recognition rate than the 3layer autoencoder, while the performance of the deep noise reduction autoencoder is improved compared with the autoencoder. The multilayer encoder network, because of its internal nonlinear structure, can better feature learning, and at the same time, the introduction of noise reduction and the
TABLE 3: Data processing sample.

\begin{tabular}{lccc}
\hline Project & Start & End & Change \\
\hline Pull-ups & 1.2 & 3.6 & 2.35 \\
$1000 \mathrm{~m}$ & 4045 & 4035 & 9.2 \\
$100 \mathrm{~m}$ & 9.6 & 8.6 & 0.7123 \\
The sitting body is bent forward & 3.5 & 7.2 & 4.1372 \\
Mental scale & 95 & 75 & 19.03 \\
Cognitive level & 7 & 16 & 9.08 \\
\hline
\end{tabular}

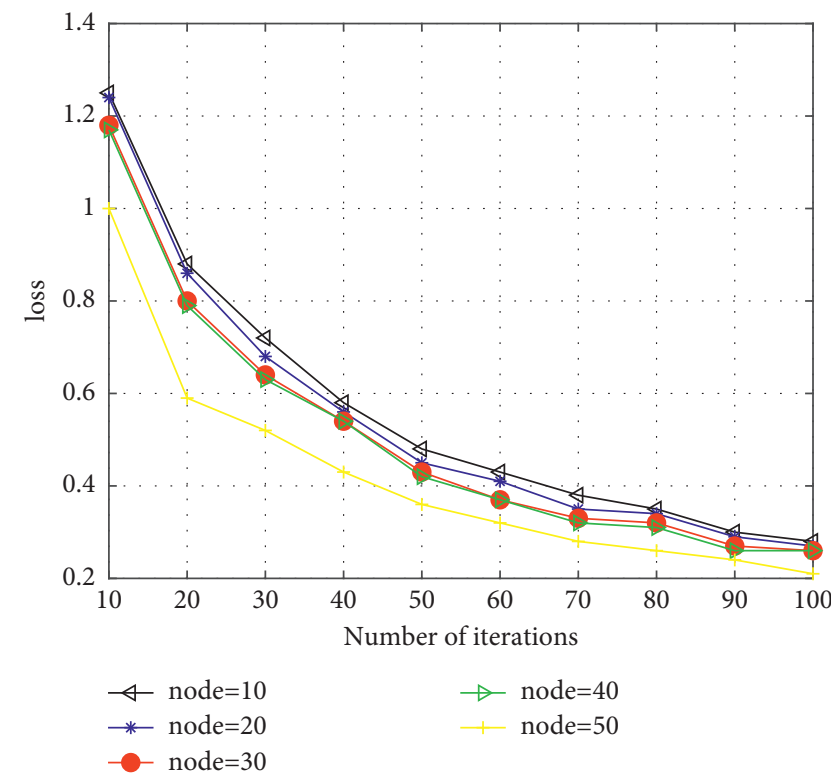

FIgURE 4: Changing curve of loss value under different nodes of hidden layers.

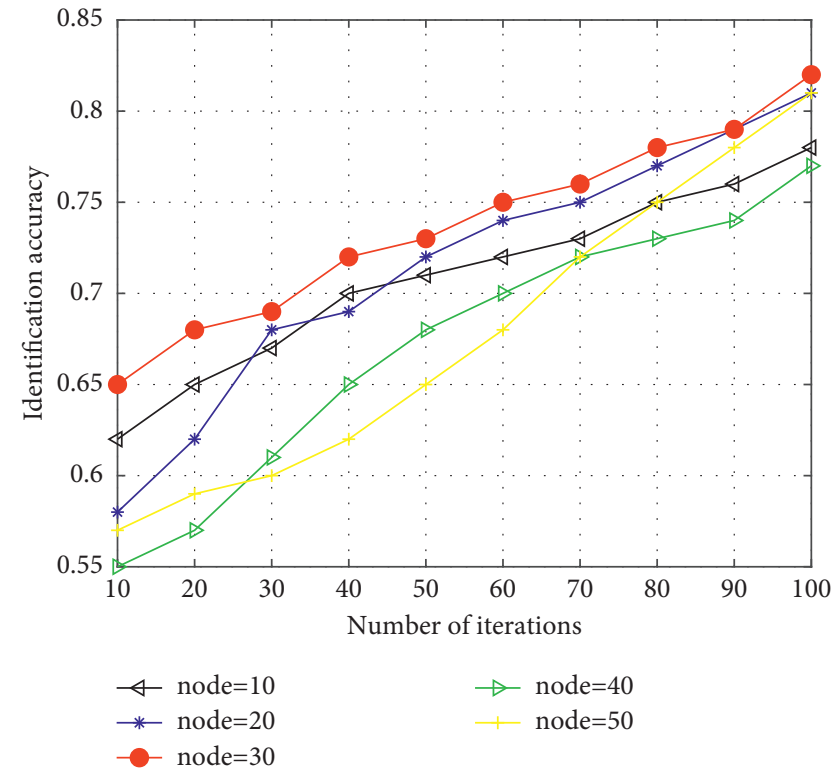

FIGURE 5: Recognition accuracy curve with different numbers of hidden layer nodes. 


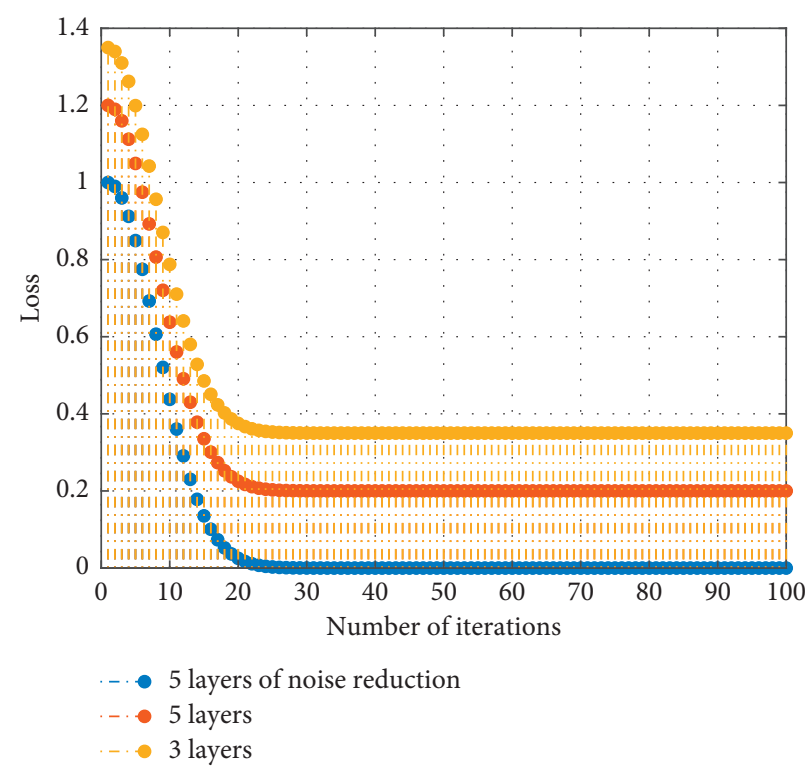

FIGURE 6: Changing curve for loss value of three autoencoders.
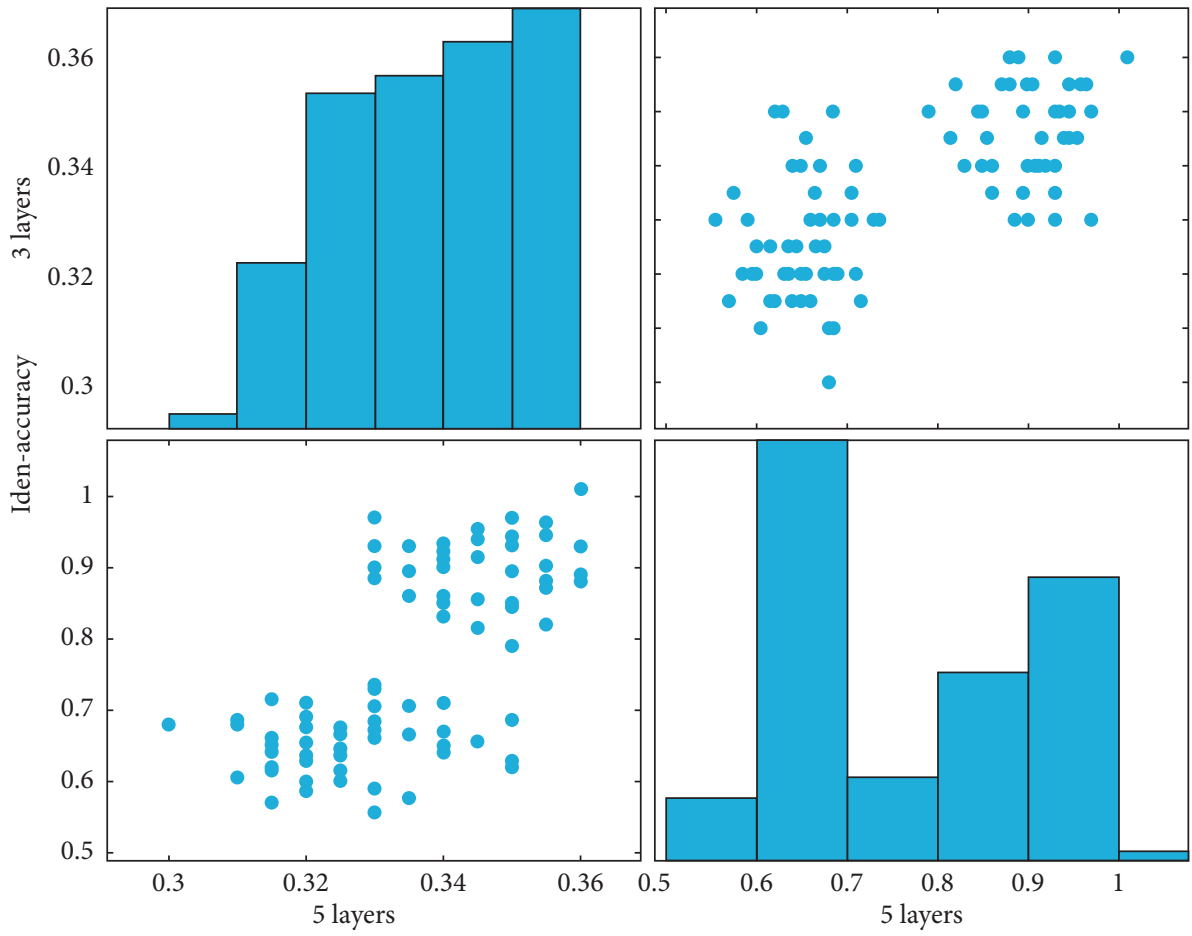

Number of iterations

FIgURE 7: Identification accuracy curve for the three autoencoders.

speech recognition results have a stronger robustness and then improve the overall system performance.

Under the same training samples and voice processing and large data of Internet of things compared with sports health management, the noise is increased artificially. The automatic encoder first trains each layer using the voice input characteristics and phonetic features from the lowdimensional ascension to high-dimensional compression. Based on the voice core characteristics, the impact is reduced for each sample. The speech recognition rate is improved by finetuning between layers using supervised training.

The systematic data analysis is mainly based on the systematic processing of the data results obtained from the various physical tests. After the query, it is presented in a visual form. People can intuitively and accurately see the physical and health status of by viewing the visual graphics. Figure 8 shows the visualization effect of the system 

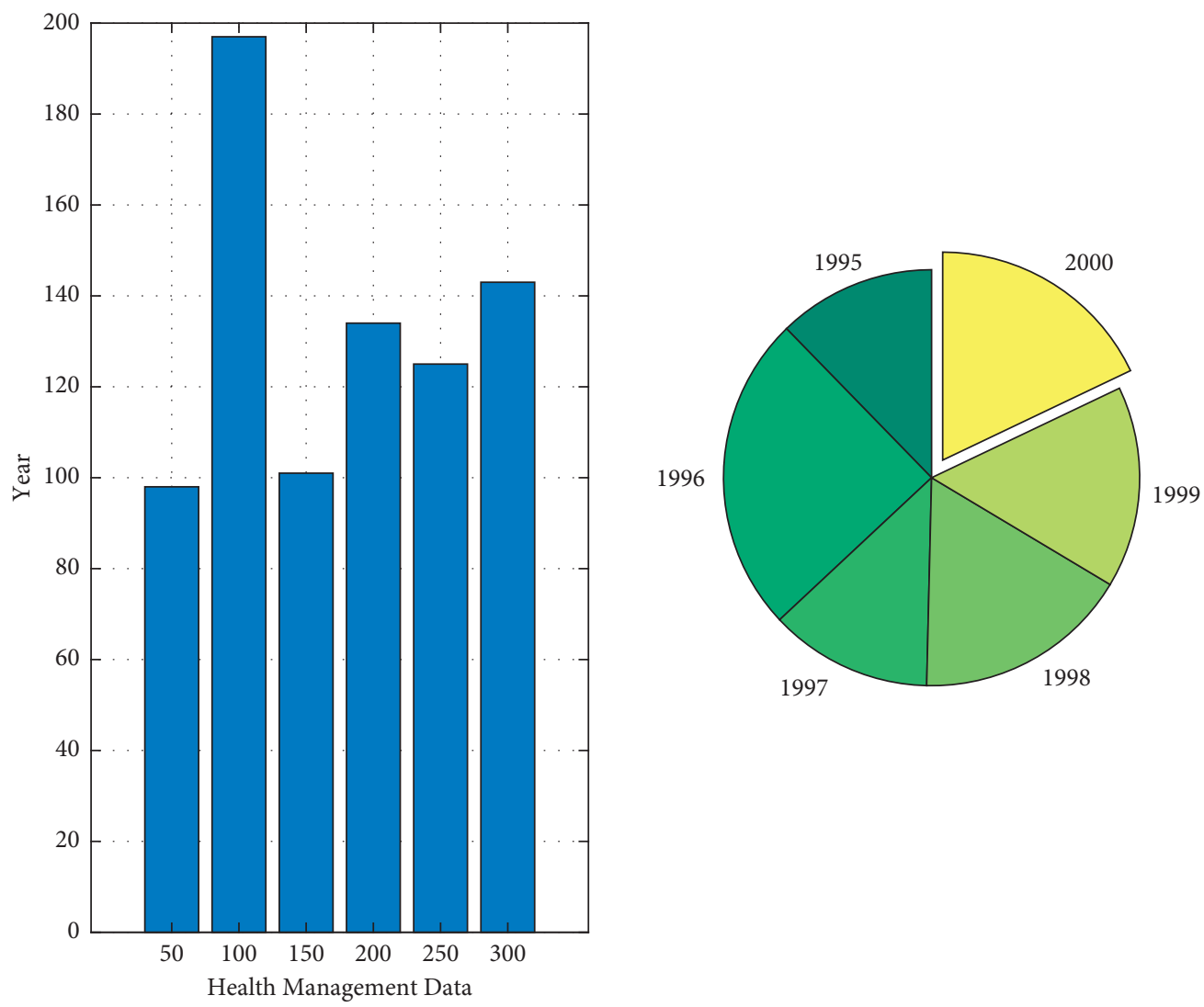

FiguRE 8: Visualization effects of big data sports and health management of voice feature processing and Internet of Things.

function, voice feature processing, and big data of sports and health management implemented by Redis technology.

\section{Conclusion}

Physical education departments should stay up with the times, conduct research into body composition and health management using voice feature processing and Internet of Things (IoT) technologies, and implement personalized management and service. The use of big data in a physique and health management system allows for high accuracy, personalization, timeliness, and efficiency in data processing and results in presentation. The sports programme should be tailored to improve the excitement and scientificity of participation in sports and exercise and ultimately to meet the goal of improving health level. The most notable feature of artificial intelligence in physique and health management is that physical quality can be tracked and evaluated in stages at one-stage intervals, and then improved fitness programmes can be provided, promoting the most efficient improvement of physique and health level and achieving the goal of physical education and teaching in schools. In future, we aim to have fog computing integrated with our scheme to improve the efficiency and accuracy even further.

\section{Data Availability}

The data used to support the findings of this study are included within the article.

\section{Conflicts of Interest}

The authors declare that they have no conflicts of interest regarding the publication of this paper.

\section{Acknowledgments}

This study was supported by People's Livelihood Research Project of Hebei Province Federation of Social Science Circles, Research on Physical Education of Improving Students' Sense of Acquisition from the Perspective of People Oriented (No. 201701936) and National Basic Scientific Research Business Expenses, Research on Social Theory Innovation of Security Development under the New Normal (Innovation Team) (No. 3142018057).

\section{References}

[1] C. Liu and Z. Zhang, "Research on the model of physical fitness and community sports of the elderly based on internet of things," RISTI - Revista Iberica de Sistemas e Tecnologias de Informacao, vol. 2016, pp. 187-197, 2016.

[2] Y. Xia and Y. Fan, "Security analysis of sports injury medical system based on internet of health things technology," IEEE Access, vol. 8, Article ID 211358, 2020.

[3] H. Panetto, P. C. Stadzisz, and W. Li, "Guest editorial: special issue on (industrial) internet-of-things for smart and sensing systems: issues, trends, and applications," IEEE Internet of Things Journal, vol. 5, no. 6, pp. 4392-4395, 2019. 
[4] Y. Ma, Y. Wang, J. Yang, Y. Miao, and W. Li, "Big health application system based on health internet of things and big data," IEEE Access, vol. 5, pp. 7885-7897, 2017.

[5] L. Research, "On the application of internet of things technology in digital sports and community fitness," Boletin Tecnico/Technical Bulletin, vol. 55, no. 4, pp. 139-145, 2017.

[6] M. G. Pecht and M. Kang, "Prognostics and health management of electronics eMaintenance," Fundamentals, Machine Learning, and the Internet of Things, vol. 10, no. 2, pp. 559-587, 2018.

[7] B. I. Mohideen and B. Assiri, "Internet of things (IoT): classification, secured architecture based on data sensitivity, security issues and their countermeasures," Journal of Information and Knowledge Management, vol. 20, no. 1, Article ID 2140001, 2021.

[8] J. Branger and Z. Pang, "From automated home to sustainable, healthy and manufacturing home: a new story enabled by the Internet-of-Things and Industry 4.0," Journal of Management Analytics, vol. 2, no. 4, pp. 314-332, 2015.

[9] B. Zheng, Z. Mei, and L. Hou, "Application of internet of things and edge computing technology in sports tourism services," Security and Communication Networks, vol. 2021, no. 3, 10 pages, Article ID 9980375, 2021.

[10] Y. Shan and Y. Mai, "Research on sports fitness management based on blockchain and Internet of Things," EURASIP Journal on Wireless Communications and Networking, vol. 2020, no. 1, pp. 121-143, 2020.

[11] H. Wang, G. Ni, J. Chen, and J. Qu, "Research on rolling bearing state health monitoring and life prediction based on PCA and internet of things with multi-sensor," Measurement, vol. 157, no. 4, Article ID 107657, 2020.

[12] J. Pang, X. Li, and X. Zhang, "Coastline land use planning and big data health sports management based on virtual reality technology," Arabian Journal of Geosciences, vol. 14, no. 12, pp. 1321-1343, 2021.

[13] L. Yao, D. Shang, and H. Zhao, "Medical equipment comprehensive management system based on cloud computing and internet of things," Journal of Healthcare Engineering, vol. 2021, no. 4, 12 pages, Article ID 6685456, 2021.

[14] J. Astill, R. A. Dara, E. D. G. Fraser, B. Roberts, and S. Sharif, "Smart poultry management: smart sensors, big data, and the internet of things," Computers and Electronics in Agriculture, vol. 170, Article ID 105291, 2020.

[15] P. A. Din, "Smart health monitoring and management system: toward autonomous wearable sensing for Internet of Things using big data analytics," Future Generation Computer Systems, vol. 91, no. 2, pp. 611-619, 2018.

[16] Y. Feng and Z. Pan, "Optimization of remote public medical emergency management system with low delay based on internet of things," Journal of Healthcare Engineering, vol. 2021, no. 1, 10 pages, Article ID 5570500, 2021.

[17] K. Anna, L. Carson, and H. Ching-Hsien, "Emerging trends, issues and challenges in Internet of Things, Big Data and cloud computing," Future Generation Computer Systems, vol. 87, no. 10, pp. 416-419, 2018.

[18] Y. Tang and D. Wang, "Optimization of sports fitness management system based on internet of health things," IEEE Access, vol. 8, pp. 91-111, 2020.

[19] A. M. Elmashtoly and C. K. Chang, "Prognostics health management system for power transformer with IEC61850 and internet of things," Journal of Electrical Engineering and Technology, vol. 15, no. 2, pp. 152-167, 2020.

[20] M. Nedunchezhiyan and A. Senthil Kumar, "Secured management using internet of things (IOT) sensing with cloud- based processing," International Journal of Scientific Research in Computer Science, Engineering and Information Technology, vol. 5, pp. 350-354, 2019.

[21] A. Sheth, H. Y. Yip, S. Shekarpour, and A. Sheth, "Extending patient-chatbot experience with internet-of-things and background knowledge: case studies with healthcare applications," IEEE Intelligent Systems, vol. 34, no. 4, pp. 24-30, 2019.

[22] M. K. Beuria, M. Saxena, and D. P. Panse, "Internet of things in healthcare -applications, issues and challenges," Solid State Technology, vol. 63, no. 6, Article ID 201103, 2020.

[23] M. M. Dhanvijay and S. C. Patil, "Internet of things: a survey of enabling technologies in healthcare and its applications," Computer Networks, vol. 153, pp. 113-131, 2019.

[24] M. Javaid and I. H. Khan, "Internet of Things (IoT) enabled healthcare helps to take the challenges of COVID-19 Pandemic," Journal of Oral Biology and Craniofacial Research, vol. 11, no. 12, pp. 83-98, 2021.

[25] P. Tiwari, "Smart waste management system for smart city based on internet of things (IoT)," Turkish Journal of Computer and Mathematics Education (TURCOMAT), vol. 12, no. 10, pp. 6-23, 2021.

[26] H. Jeong, J. H. Shen, and B. Ahn, "A study on smart healthcare monitoring using IoT based on blockchain," Wireless Communications and Mobile Computing, vol. 2021, no. 2, 9 pages, Article ID 9932091, 2021.

[27] A. Ali, "Alshmrany. Health monitoring and management system by using wireless sensor network and internet of things (IoT)," International Journal of Computer Network and Information Security, vol. 19, no. 12, pp. 179-184, 2019. 